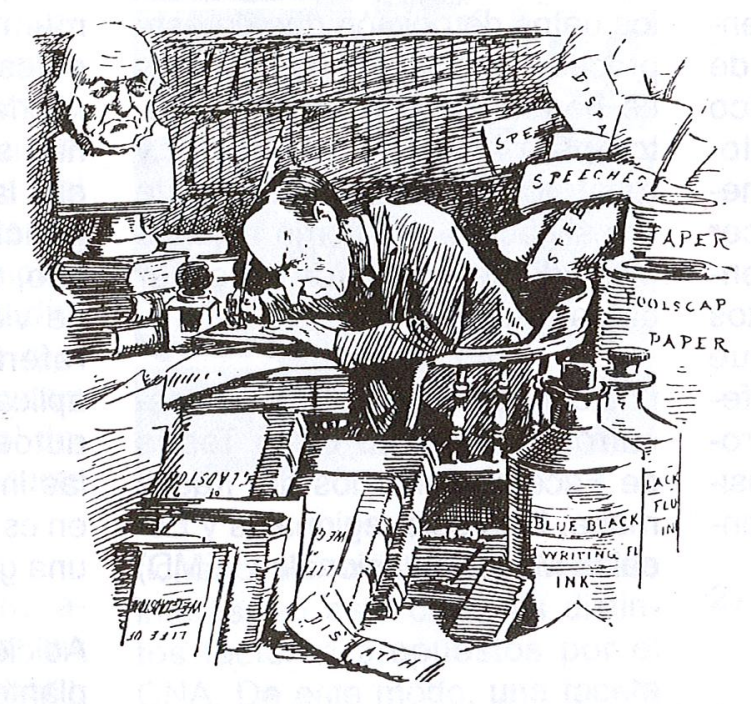

\title{
TEORÍA DE FACETAS: GUÍA METODOLÓGICA PARA LA RECOLECCIÓN DE OPINIONES DENTRO DEL PROCESO DE AUTOEVALUACIÓN INSTITUCIONAL
}

Pablo Páramo Ps. M.Sc. Profesor Asociado Universidad Pedagógica Nacional

El propósito general del presente artículo es presentar los principios básicos que orientan el trabajo investigativo a partir de la Teoría de Facetas, con el cual se muestra al los investigadores la manera de ser más sistemáticos en la definición de las hipótesis y en la operacionalización de las variables que pretenden estudiar. Específicamente, la aplicación del modelo de Facetas se ilustra en el proceso de recolección de información que se requiere den- tro de la autoevaluación institucional con fines de Acreditación, de los programas académicos a nivel de pregrado.

El Consejo Nacional de Acreditación ha establecido los lineamientos para la acreditación de los programas académicos de educación superior con miras a fortalecer la calidad de educación superior y hacer reconocimiento público del logro de altos niveles de calidad (CNA, 1998). Dentro del proceso de acreditación, 'distingue tres etapas, una de las cuales se refiere a la autoevaluación, que consiste en el estudio que deben llevar a cabo los programas académicos con la participación amplia de la comunidad académica sobre la base de los criterios, características variables e indicadores definidos por el mismo CNA. Sin embargo, el proceso puede resultar muy complejo si no se cuenta con una metodología que permita guiar 
las observaciones y evaluar las relaciones entre los distintos aspectos sobre los que se solicita información.

La Teoría de Facetas se presenta como un modelo teórico, de investigación y metodológico orientado a llenar este vacío. Esta metateoria provee una metodología que permite conocer cómo se da la interrelación entre las opiniones de los distintos estamentos del programa que está siendo evaluado, los diferentes criterios y variables propuestas en cada factor e inclusive si se desea, entre los distintos factores.
La Teoría de Facetas permite integrar el dominio de interés de la investigación, en este caso la autoevaluación con fines de acreditación, con el análisis de los datos de opinión que de este proceso se deriven, ya que ofrece herramientas y procedimientos para analizar, estructurar y reestructurar los contenidos de investigación, así como algunos procedimientos para procesar datos multivariados.

Los conceptos y las técnicas desarrollados dentro de la Teoría de Facetas como los de: frases mapa, hipótesis regionales y Escalas MultiDimensionales (EMD) han permitido el desarrollo de la investigación científica por la oportunidad que ofrece la teoría de dar una nueva mirada a la investigación sobre el comportamiento. Shye y Elizur (1994) muestran cómo las técnicas derivadas de la Teoría de Facetas han sido utilizadas ampliamente en la investigación sobre inteligencia, comunicación, mercadeo, salud, educación y calidad de vida. El presente artículo se referirá específicamente a su aplicación dentro del proceso de autoevaluación que adelantan las instituciones universitarias, y en esa medida se presenta como una guía de trabajo investigativo.

Adicionalmente, la teoría ha replanteado el rol de la estadística en la investigación del comportamiento. A diferencia de la estadística tradicional, las observaciones no son aisladas o vistas como fenómenos aislados. Por el contrario, las variables objeto de observación se ven como parte de un continuo del comportamiento. Esta metateoría busca integrar tres componentes de investigación según Guttman $\left(1982^{a}\right)$ : una hipótesis de correspondencia entre un sistema definitorio para un universo de observaciones, un aspecto de la estructura empírica de dichas observaciones y una racionalidad para tal hipótesis. La Teoría de Facetas ajusta su filosofía con herramientas prácticas para las distintas fases de la exploración científica: definición de los contenidos de investigación, la formulación de hipótesis, diseño de cuestionarios y análisis de datos multivariados. Se establece la necesidad de la investigación empírica y la necesidad de 
la estadística pero teniendo presente que antes que la cuantificación sin sentido, es necesario la comprensión y definición exacta de lo que está siendo estudiado. Por lo tanto, como anota Donald (1995), es el conocimiento y el entendimiento sustantivo de la disciplina lo que ayudará a formular la definición precisa del universo de lo que se pretende investigar, siendo la definición precisa del objeto de estudio, más que la estadística, lo que contribuye de forma significativa a la ciencia acumulativa y al desarrollo de leyes de la conducta humana.

La Teoría de Facetas define apriori un sistema para guiar las observaciones (Canter, 1985b). Para el caso del presente trabajo, las observaciones se refieren al conjunto de opiniones manifestadas por los distintos estamentos universitarios (directivos, profesores, estudiantes) y pares académicos sobre el programa académico que está siendo evaluado. Esto supone la definición de los límites del área de investigación de manera precisa para que sirva de guia en el trabajo del investigador.

El punto de partida del investigador es especificar exactamente lo que comprende su área de interés. Lo primero que se hace en teoría de facetas es definir el universo de contenido, identificando los principales componentes conceptuales de tal dominio a través de las facetas. Esas facetas prescribirán precisamente los límites de la investigación, définen el universo de contenido y se usan directamente para generar preguntas de un cues-

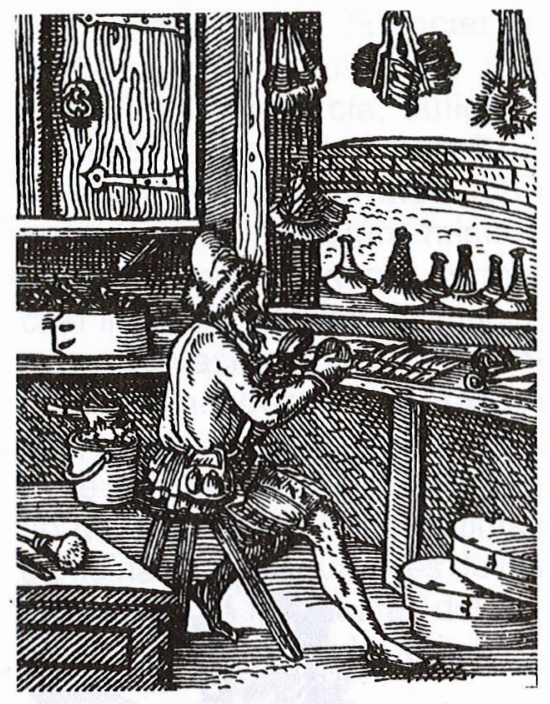

tionario acerca del fenómeno de interés; en este caso los distintos factores propuestos por el CNA. De este modo, una faceta es una categorización conceptual que fundamenta un grupo de observaciones (Brown, 1985).

Las facetas de la autoevaluación en nuestro caso corresponden a los Factores de: procesos académicos, bienestar, egresados e impacto y recursos físicos y financieros. Aquí el intento es el de identificar los principales componentes del dominio de interés y no el de encontrar preguntas especificas que puedan ser incluidas en la evaluación.

Hay dentro del modelo tres tipos de facetas:

\section{1) Las facetas de contexto} describen lo que puede ser considerado el contexto del estudio o sus parámetros de población. Por ejemplo, edad y género podrian considerarse como facetas de contexto en la medida que describen caracteristicas de los partici- pantes de un estudio. Dentro del proceso de auto-evaluación institucional una faceta de contexto corresponde al interés de estudiar diferencias entre los roles que se observan en las distintas instituciones universitarias (profesores, estudiañtes y directivos). De esta manera las facetas de Contexto se formulan cuando se desea explorar diferencias individuales 0 situacionales en relación con el dominio del estudio, por ejemplo diferencias entre roles o funciones dentro de la institución.

2) Las facetas de dominio describen lo que podría considerarse el cuerpo del área de interés. Por ejemplo, dentro del proceso de autoevaluación, las facetas que describen lo que está siendo evaluado se refieren a los Factores y los Criterios, los cuales pueden evaluarse en relación con alguna faceta de interés particular como el PEI institucional, la cual podría considerarse la faceta foco o central dentro de las de dominio (Donald 1995).

3) Las facetas de Rango describen las posibles respuestas ante los estímulos suministrados por las facetas de dominio: la respuesta de la población (descrita por las facetas de contexto) ante el dominio (descritas por las facetas de dominio). Cuando el rango de cada ítem es ordenado, y ordenado en el mismo sentido (es decir tiene el mismo significado en la base) 
éste es considerado un Rango Común. Como el rango común define las posibles respuestas para el dominio, esta es una faceta importante para definir la naturaleza y definición del área de estudio. Para nuestro ejemplo, el rango común tendría que ver con el grado de acuerdo con diferentes afirmaciones que se deriven de las facetas bajo estudio. El rango común sobre el cual se recogerían las opiniones estaría dentro de una escala Likert de cinco puntos, que iría desde: Muy en Desacuerdo a Totalmente de Acuerdo, o dependiendo del Factor que se evalúe podría ir desde: Muy Insatisfecho a Totalmente Satisfecho.

Las facetas están constituidas por elementos que corresponden a los diferentes valores que describen las variaciones de las facetas. Directivos del programa, profesores, estudiantes y pares académicos, por ejemplo, son los elementos constitutivos de la Faceta «Estamento Universitario»; la existencia, conocimiento, aplicabilidad, coordinación, idoneidad, suficiencia, son elementos de la Faceta Criterio; etc.

Dentro de esta aproximación, cada faceta y sus elementos deben ser mutuamente excluyentes en relación con las otras facetas y sus elementos. Deben ser exhaustivas en el sentido de que los elementos deben comprender todos los componentes definitorios de la faceta; en otras palabras, deben ser exhaustivas de los aspectos del dominio de interés. Por este motivo, cada faceta y sus elementos constituti-

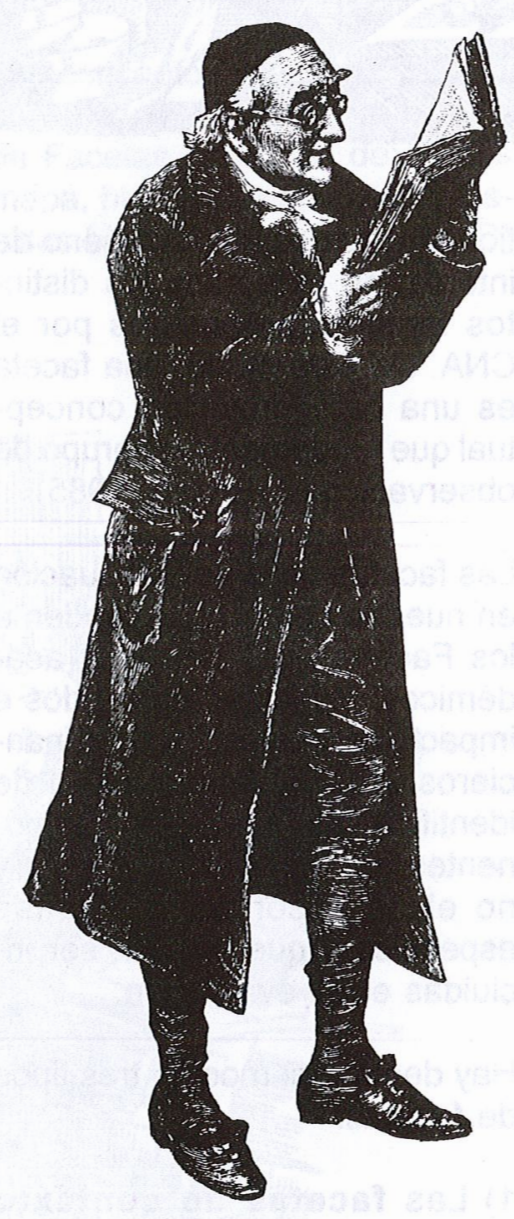

Una vez que se han identificado las facetas de Contexto, del Dominio y de Rango, éstas se vinculan a través de una Frase Mapa la cual se define según Shye (1978) como una afirmación verbal del dominio y del rango de un mapeo incluyendo conectores entre las facetas a través del lenguaje ordinario. Una frase mapa es una forma concisa de especificar un universo de contenido: los componentes del dominio de investigación y la relación entre ellos, lo cual define un conjunto de hipótesis que requieren ser verificadas.

Así, el modelo se puede ajustar perfectamente para una evaluación institucional con la ventaja de poder contar de antemano con casi la totalidad de las facetas y los elementos ya que éstos estarían dados en los Lineamientos para la Acreditación del CNA (1998). Para definir las facetas y sus elementos constitutivos habría que definir qué tipo de facetas de contexto podrían ser relevantes para el estudio: ¿el género de los participantes?, ¿su rol o función dentro de la institución?, ¿su nivel educativo? Para este caso, resulta bastante sencillo definir los elementos. Sin embargo, para definir las facetas de dominio 
será necesario hacer una revisión cuidadosa de la literatura sobre los lineamientos del Consejo Nacional para la Acreditación (CNA, 1998).

Mientras tanto, propongamos a manera de ejemplo una gran frase mapa, para la cual la Facetas de Contexto estaría integrada por los elementos: Directivos, Profesores, Estudiantes y Pares Académicos; las de Dominio pueden estar integradas por las facetas: Factores con sus elementos: procesos académicos, bienestar, egresados e impacto, recursos físicos y financieros; Criterios, constituida por sus elementos: eficiencia, suficiencia, calidad, etc; y la faceta Propósitos Institucionales, con sus elementos: visión, misión, objetivos, medio externo, formación integral, relación de funciones, evaluación y gestión y comunidad académica. La faceta de Rango Común podría definirse a partir del Grado de Acuerdo que se asocia a las distintas combinaciones resultantes de los elementos de las facetas del dominio: desde «Muy de Acuerdo» pasando por distintos grados, hasta «Muy en Desacuerdo»; esta escala de 5 o 7 grados definiría los elementos para esta faceta.

De esta manera, la frase mapa debe dar origen a las posibles afirmaciones $u$ observaciones que deben realizarse a partir de la definición del estudio. En la siguiente frase mapa se está definiendo el universo de observaciones y los límites de la investigación sobre el proceso de evaluación:

En qué medida la persona $(x)$, que hace parte del ESTAMENTO UNIVERSITARIO

evalúa el CRITERIO

$$
\begin{aligned}
& \text { 1. existencia } \\
& \text { 2. conocimiento } \\
& \text { 3. aplicabilidad } \\
& \text { 4. coordinación } \\
& \text { 5. idoneidad } \\
& \text { 6. suficiencia }
\end{aligned}
$$

\section{RANGO COMÚN \\ 5. Completamente de acuerdo \\ 4. De acuerdo \\ 3. Medianamente de acuerdo \\ 2. En desacuerdo \\ 1. Totalmente en desacuerdo}

Una frase mapa como la presentada arriba sirve de guía para delimitar el dominio de todo el proceso que orienta las obser- del

\section{FACTOR}

1. Directivos

2. Profesores

3. Estudiantes

4. Pares académicos

5. Egresado
1. Procesos académicos
2. Bienestar
3. Organización y administración
4. Egresados e impacto
5. Recursos físicos y financieros

en la consecución de los PROPÓSITOS INSTITUCIONALES vaciones centradas en apreciaciones $u$ opiniones de parte de los distintos estamentos académicos. Sin embargo, si se quiere estudiar con mayor detalle cada factor, será necesario construir una frase mapa para cada uno. A continuación, se presenta un 
frases mapa que puede igualmente orientar el proceso de recolección de opiniones en caso de que se adopte la perspectiva de crear una frase mapa por factor:

FACTOR: BIENESTAR INSTITUCIONAL ${ }^{1}$

(C)

En qué medida la persona del...

ESTAMENTO UNIVERSITARIO considera que hay CRITERIO sobre...

\author{
Profesor \\ Estudiante \\ Directivo
}

1. existencia
2. conocimiento
3. aplicabilidad
4. coordinación
5. idoneidad
6. suficiencia

(P)

(B)

BIENESTAR INSTITUCIONAL... que contribuyen al... PEI

1. Políticas de Bienestar

2. Oficina de Bienestar

3. Servicios que presta

4. Actividades formativas extracurriculares

5. Profesionales que prestan los servicios

6. Clima institucional

\author{
1. Misión \\ 2. Visión \\ 3. Objetivos \\ 4. Medio externo \\ 5. Form. integral \\ 6. Rel. de funciones \\ 7. Evaluación y gestión \\ 8. Comunidad académica
}

\section{RANGO COMÚN}

1. Totalmente insatisfecho

2. Insatisfecho

3. Medianamente satisfecho

4. Satisfecho

5. Totalmente satisfecho

De las frases mapa se derivan un conjunto de afirmaciones resultado de las múltiples combinaciones entre los distintos elementos que configuran las facetas del dominio de interés. A estas distintas combinaciones se les denomina perfiles; el conjun- to de estos perfiles da origen al cuestionario, el cual deberá suministrarse a los distintos estamentos identificados en la faceta. El número de perfiles resulta de multiplicar el número de elementos de las facetas de dominio, enmarcando así el dominio del estudio y la guía para hacer las preguntas o afirmaciones correspondientes a las observaciones que conformarán el cuestionario. Se puede repetir perfiles dependiendo el interés que se tenga en explorar algunas combinaciones en particular. Cada

Para el caso de la autoevaluación institucional se propone tomar el PEI como faceta foco contra la cual se evalúen los demás factores. 
perfil o afirmación derivada de la frase mapa se evalúa sobre el Rango Común que generalmente es una escala tipo Likert .

A continuación se presentan algunos perfiles derivados de la

\begin{tabular}{|c|c|c|}
\hline PERFIL & AFIRMACIÓN & $\begin{array}{c}\text { ESCALA } \\
\text { TI-I-MS-S-TS }\end{array}$ \\
\hline C2B3P3 & $\begin{array}{l}\text { Conozco las actividades deportivas y los servi- } \\
\text { cios de salud que ofrece la U.P.N. y creo que } \\
\text { éstos contribuyen al desarrollo intelectual, éti- } \\
\text { co, físico y socioafectivo de los estudiantes. }\end{array}$ & 12345 \\
\hline С6B3P.3 & $\begin{array}{l}\text { Los servicios de bienestar de la U.P.N. son su- } \\
\text { ficientes para atender las necesidades de los } \\
\text { estudiantes. }\end{array}$ & 12345 \\
\hline C6B4P5 & $\begin{array}{l}\text { Las actividades culturales desarrolladas por } \\
\text { Bienestar Universitario son suficientes para la } \\
\text { formación integral de los estudiantes. }\end{array}$ & 12345 \\
\hline C5B5P3 & $\begin{array}{l}\text { El personal profesional que presta los servi- } \\
\text { cios de bienestar en la universidad es idóneo } \\
\text { para atender las necesidades que tienen los } \\
\text { estudiantes. }\end{array}$ & 12345 \\
\hline C1B3P1 & $\begin{array}{l}\text { Los servicios de bienestar, en cuanto a recur- } \\
\text { sos bibliográficos y audiovisuales, contribuyen } \\
\text { a que el Programa de Educación Especial lo- } \\
\text { gre su visión en las dimensiones de docencia } \\
\text { e investigación. }\end{array}$ & \\
\hline
\end{tabular}

\section{Método de análisis de datos}

La Teoría de Facetas tiene un conjunto de procedimientos analíticos asociados, amétricos y multivariados, denominados «Escalamiento Multidimensional». Estos se refieren a un grupo de modelos en los que la información contenida, en este caso los ítems, se representa como un conjunto de puntos en el espacio. Para analizar los datos producto del cuestionario se prepara una matriz que contiene las respuestas de los participantes del estudio, colocando a las personas en las filas y los ítems del
Frase Mapa presentada anteriormente, correspondiente al Factor: Bienestar institucional, con sus respectivas afirmaciones:

$x^{2}$


tad de Educación de la U.P.N. Como puede observarse, en la gráfica fue posible establecer cinco particiones o regiones correspondientes a cada uno de los factores definidos dentro de la faceta: Factor. En los gráficos se observa si se representan los juicios de los participantes sobre el estudio de interés a partir de las estructuras hipotetizadas. Lo primero que hay que hacer notar es que los ítems no están distribuidos aleatoriamente sino que en el plano seleccionado es posible hacer unas particiones en regiones, de tal suerte que en cada una de ellas se encuentran solamente, o casi solo, los ítems que pertenecen a un elemento de la faceta propuesta. Así, al analizar las facetas, se espera que las hipótesis se relacionen con la manera como las variables se muestren geométricamente, o de otra manera, las hipótesis de facetas predicen cómo se verán los datos (empíricos de acuerdo con la frase mapa) cuando éstos sean representados sobre el papel.

El primer tipo de hipótesis que se deriva de la teoría de facetas afirma la existencia de las regiones de contigüidad de los elementos de cada faceta. Si la hipótesis establece que deben surgir para una de las faceta cinco regiones correspondientes a cada uno de los cinco elementos formulados en una faceta de la frase mapa, una región debería incluir todas las variables (perfiles o ítems) relacionadas con el primer elemento, otra región para el segundo y así sucesivamente. Esta contigüidad implica que cada elemento de una faceta estará representado por una región separada dentro de una configuración geométrica y todos los perfiles en cada región son del mismo componente de ese elemento. De la misma manera se debe proceder para analizar cada faceta propuesta en la frase mapa.

Figura No. 2. Autoevaluación en el programa de educación especial. Faceta: Factor.

Los números corresponden a los ítems del cuestionario. En este caso los ítems se pudieron agrupar en cinco regiones correspondientes a los elementos constituyentes de la faceta.

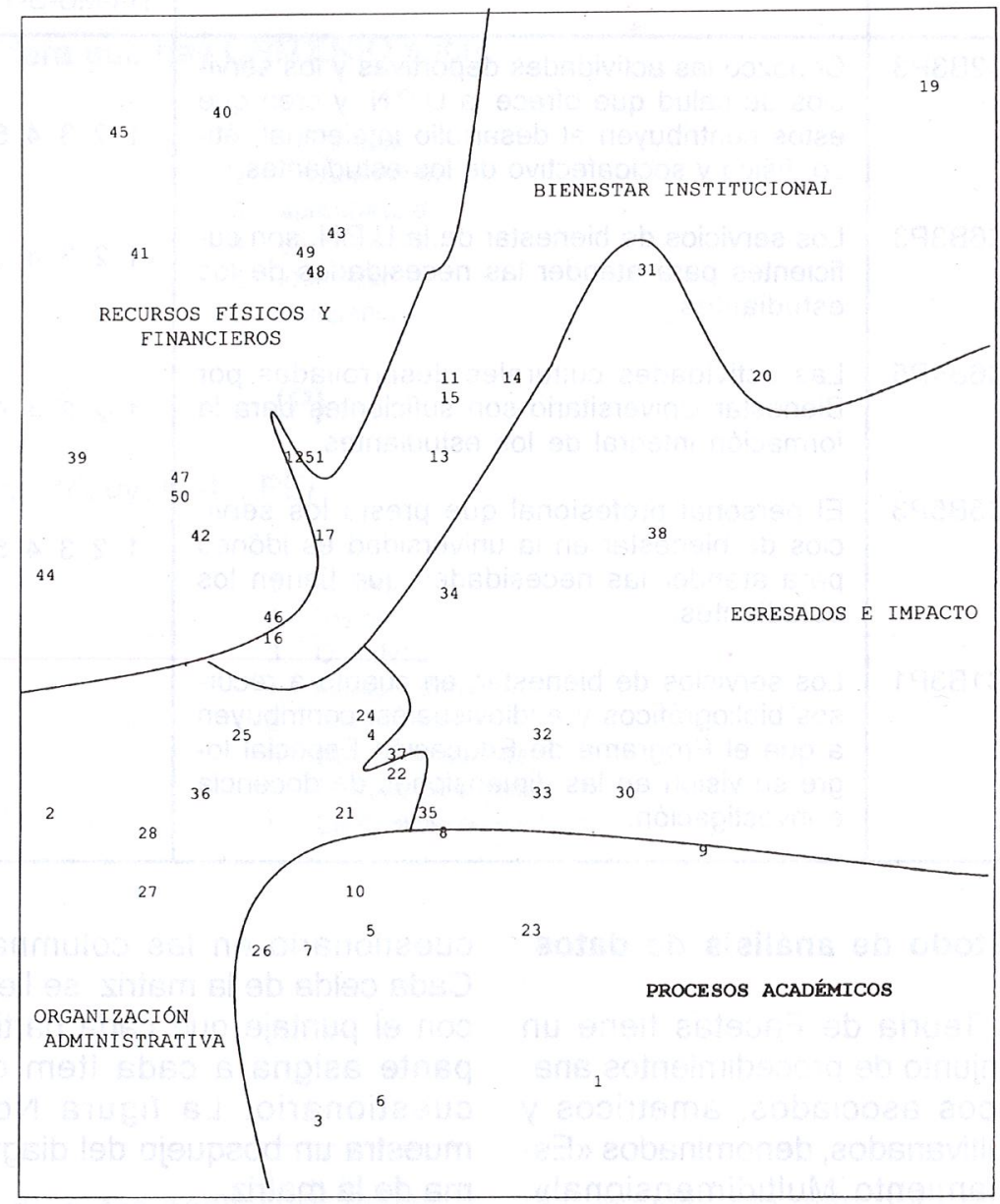

Como se mencionó anteriormente, también es posible analizar cada Factor propuesto por el C.N.A. como una faceta diferente y de esta forma obtener información más detallada de cada uno de ellos. Si se opta por esta posibilidad, deberá crearse una frase mapa para cada factor. La
Figura No. 3 muestra los resultados hipotéticos de la faceta Bienestar Institucional presentada arriba, con sus elementos: Políticas de Bienestar Oficina de Bienestar, Visión, Servicios que presta, Actividades formativas extra-curriculares, Profesionales que prestan los servicios y Cli- 
ma Institucional. EI mapa SSA se interpreta con base en la existencia o no de regiones significativas desde cualquiera de las dimensiones, de acuerdo con la estructura de las facetas propuestas en la frase mapa. Con este análisis para cada faceta se busca ver si éstas y la frase mapa tienen evidencia empírica dependiendo si se encuentran o no las regiones de contigüidad para cada elemento. De esta manera se analiza si los elementos propuestos para cada faceta tienen una representación para los participantes del estudio y si son predictores del factor que está siendo evaluado. Una vez que se analiza la estructura de cada una de las facetas, se procede a ana-

Figura No. 3. Autoevaluación institucional: Faceta: Bienestar institucional.

Los números corresponden a los ítems del cuestionario. Obsérvese que se agrupan en cinco regiones diferentes con lo cual se da evidencia empírica a la faceta hipotetizada.

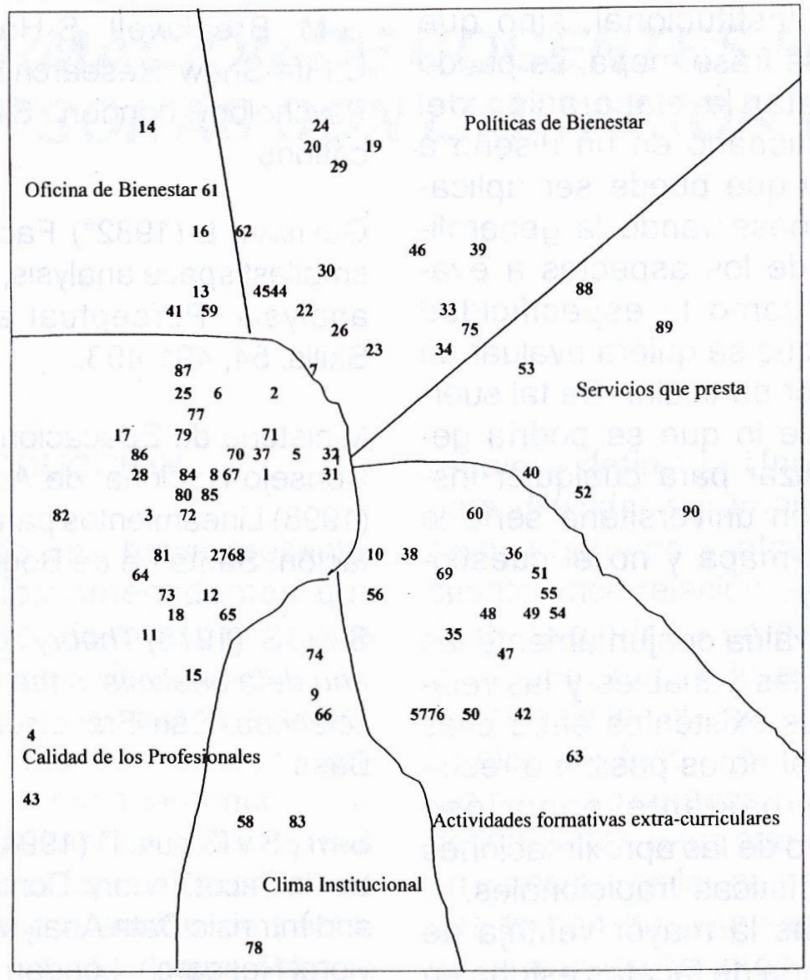

Para resumir, el proceso de construcción de instrumentos de opinión para la autoevaluación institucional a partir de la teoría de facetas establece los siguientes pasos:

1. Seleccione los indicadores en los que se solicite recoger información sobre opiniones o apreciaciones de los distintos estamentos académicos.

2. Identifique las fuentes o estamentos académicos de los cuales se solicita información para cada indicador teniendo presente las variables sobre las que se solicita información. De esta manera defina su(s) faceta(s) de contexto (profesores, directivos, pares académicos, etc.).

3. A partir de los criterios con que se debe evaluar, defina su faceta criterio y los elementos que le corresponden (transparencia, pertinencia, correspondencia, etc.).

4. Para cada faceta de factor defina los elementos constitutivos a partir de las características y variables.

5. De acuerdo con las facetas de dominio seleccione la faceta de rango común (satisfacción, acuerdo, importancia o conocimiento).

6. Escriba la frase mapa que conecte las facetas.

7. Defina únicamente los perfiles que se solicitan.

8. Diseñe el instrumento: Redacte las afirmaciones que se derivan de los perfiles. Tenga en cuenta que se pueden redactar varias afirmaciones por perfil a partir de los distintos matices que pueden tomar los elementos de las facetas. 
9. Busque la confiabilidad de su cuestionario con sus colegas.

10. Valide finalmente el instrumento con una muestra pequeña de la población.

11. Procese los datos empíricos a través del uso del SSA.

13. Interprete los hallazgos y observación dependiendo de si los resultados permiten soportar o rechazar la estructura hipotética o relaciones entre las facetas de las frases mapa.

Para terminar, se presentan a continuación las principales ventajas de la Teoría de Facetas dentro del proceso de autoevaluación:

1. Permite hacer una definición precisa del universo de contenido u observaciones.

2. Especifica instrucciones acerca de cómo hacer las observaciones y define la manera de hacer las preguntas para un cuestionario.

3. Se constituye en una ayuda para la percepción de relaciones sistemáticas.

4. Permite agregar y suprimir facetas del contenido del contexto o del dominio.

5. Facilita la validación de teorías y de hipótesis.

6. Ayuda a desarrollar de investigación acumulativa en la medida que se validan o rechazan las facetas propuestas.

7. Permite construir escalas de evaluación, tomando los principales elementos dentro de un universo posible de observaciones. Evita la arbitraria construcción de escalas de evaluación sin un soporte teórico, sin poder determinar la estructura de cada uno de sus componentes. Así, para los propósitos de cualquier estudio, en lugar de definir a priori un conjunto de afirmaciones para un cuestionario de forma arbitraria, lo que se hace es definir el conjunto de facetas del dominio de interés.

8. Evita generar múltiples cuestionarios para evaluar los distintos aspectos solicitados por el C.N.A. y facilita la generalidad de los hallazgos. Los intentos previos para producir instrumentos de evaluación han fallado porque, como dice Donald (1983), no son lo suficientemente generales para ser utilizados ampliamente en cualquier situación, y cuando lo son, su utilidad es limitada para una situación específica. La teoría de facetas, puede mostrar no sólo los vacíos en la evaluación institucional, sino que con la frase mapa, se puede orientar la elaboración del cuestionario en un diseño a priori que puede ser aplicado conservando la generalidad de los aspectos a evaluar como la especificidad con que se quiera evaluar un Factor particular. De tal suerte que lo que se podría generalizar para cualquier institución universitaria sería la frase-mapa y no el cuestionario.

9. Se evalúa conjuntamente las distintas variables y las relaciones existentes entre ellas lo cual no es posible o resultaría bastante engorroso dentro de las aproximaciones estadísticas tradicionales.

10. Quizás la mayor ventaja de la teoría de facetas estriba en ser un método heurístico con posibilidad de construcción teórica que abre posibilidades para explorar empíricamente relaciones complejas entre variables. Gracias al hecho de no estar ligada a ningún ámbito investigativo específico, ofrece la oportunidad de considerar cualquier área de investigación.

\section{REFERENCIAS}

Brown, J (1985) An introduction to the uses of facet theory. En: D. Canter (Ed), Facet Theory: Aproaches to sojcial research. New York. Springer.

Canter, D. (1985b) (ed) Facet Theory: Approaches to Social Research. New York: SpringerVerlag.

DonALD, I.J. (1995) Facet Theory: Defining Research Domains. En: G.M. Breakwell, S. Hammond y C.Fife-Shaw: Research Methods in Psychology. London. SAGE Publications.

GutTMAN, L (1982 ${ }^{a}$ ) Facet Theory, smallest space analysis, and factor analysis. Perceptual and Motor Skills. 54, 491-493.

Ministerio de Educación NacionalConsejo Nacional de Acreditación (1998) Lineamientos para la acreditación. Santa Fe de Bogotá,

SHYE, S. (1978) Theory construction and data analysis in the behavioral sciences. San Francisco: JosseyBass.

SHYE, S y ElIzUR, D (1994) Introduction to Facet Theory: Content Design and Intrinsic Data Analysis in Behavioral Research. London SAGE. 